\title{
The Mathematical Modelling of Heat Transfer in Electrical Cables
}

\author{
Andrej Bugajev (Researcher, Vilnius Gediminas Technical University - VGTU), \\ Gerda Jankevičiūtè (Researcher, VGTU), Olga Suboč (Researcher, VGTU), \\ Natalija Tumanova (Researcher, VGTU)
}

\begin{abstract}
This paper describes a mathematical modelling approach for heat transfer calculations in underground high voltage and middle voltage electrical power cables. First of the all typical layout of the cable in the sand or soil is described. Then numerical algorithms are targeted to the two-dimensional mathematical models of transient heat transfer. Finite Volume Method is suggested for calculations. Different strategies of nonorthogonality error elimination are considered. Acute triangles meshes were applied in two-dimensional domain to eliminate this error. Adaptive mesh is also tried. For calculations OpenFOAM open source software which uses Finite Volume Method is applied. To generate acute triangles meshes aCute library is used. The efficiency of the proposed approach is analyzed. The results show that the second order of convergence or close to that is achieved (in terms of sizes of finite volumes). Also it is shown that standard strategy, used by OpenFOAM is less efficient than the proposed approach. Finally it is concluded that for solving real problem a spatial adaptive mesh is essential and adaptive time steps also may be needed.
\end{abstract}

Keywords - Power transmission; Numerical models; Finite element analysis; Finite volume methods; Convergence of numerical methods.

\section{INTRODUCTION}

This research is aimed to get closer to develop design rules for power transmission lines and cables, which have to meet the latest power transmission network technical and economical requirements. At present the power lines are overdimensioned by up to $60 \%$ in terms of transmitted power. However, today, as the new distributed generating capacities are installed e.g. large wind farms, bio-gas plants or waist-toenergy plants, the infrastructure of power grid must be redesigned or new optimization strategies for the available grid developed. Power cables for power distribution applications are still rated according to IEC 287 and IEC 853 standards, which use the Neher and McGrath methods from 1957 [1]. However, today there are many applications where analytical and heuristic formulas cannot describe precisely enough the conditions under which the cables are installed. An example could be an underground cable route, where the installation conditions for a cable are different only for a short distance (crossing the road). The present standards require that the cable's current-carrying capacity must be reduced only due to the fact that the cables route crosses the road. To be on the safe side this rule is acceptable, but today the cost effective designing of cable installations comes first as the copper price level has reached its maximum since decades.
The knowledge of dynamics (in time) of heat distribution in/around electrical cables is necessary to optimize the usage of electricity transferring infrastructure. It is important to determine: maximal electric current for the cable; optimal cable parameters under certain circumstances; cable life expectancy; other engineering factors.

Fundamentals of the heat distribution in cables are given [2], but for further readings refer [3], [4] and [5]. In papers [6] and [7] efficient parallel numerical algorithms for simulation of temperature distribution in electrical cables are presented and inverse problem for fitting the diffusion coefficient of the air-isolation material mixture to the experimental data are solved.

Numerical algorithms for parabolic and elliptic problems with discontinuous coefficients have been widely investigated in many papers. The use of standard finite element method (FEM) to solve interface problems is equivalent to arithmetic averaging of discontinuous coefficients. The mixed FEM leads to the harmonic averaging if special quadrature formulae are used - see, e.g. works [8] and [9].

Conservative finite-difference schemes for approximation of parabolic and elliptic problems were derived by Tikhonov/Samarskii in [10] and [11]. These schemes are robust and use only general assumptions on the position of the interface. Also such finite difference schemes were proposed, which approximate with the second order of accuracy not only the solution, but also the normal flux through the interface see [12] and [13] for details.

\section{II.PROBLEM FORMULATION}

The main aim of the research is to develop and validate a set of mathematical models and numerical algorithms for the heat transfer simulation in underground electrical cables (see Fig. 1 and Fig. 2).

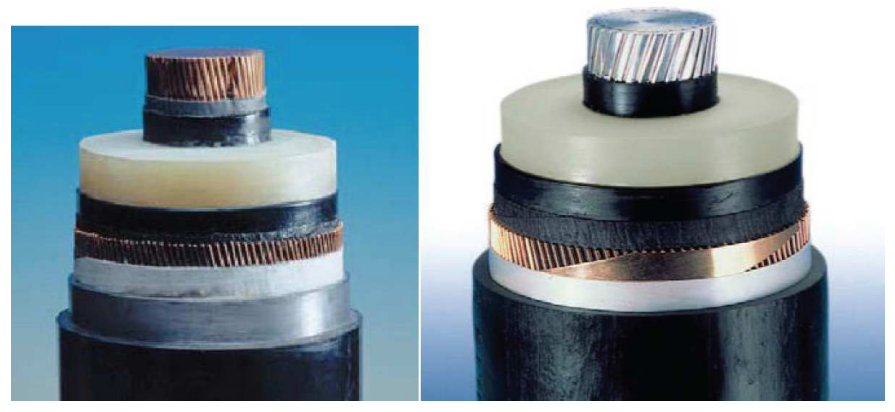

Fig. 1. Typical high-voltage $(110 \mathrm{kV})$ cables. 


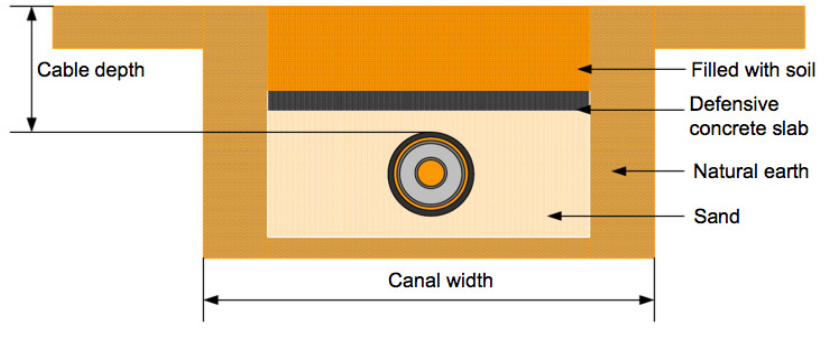

Fig. 2. Underground layout in canal.

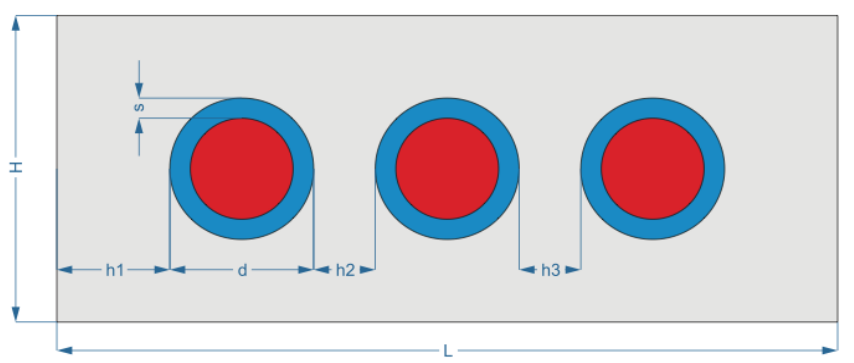

Fig. 3. An example of geometry.

Since the heat transfer mechanism in the underground electrical cables is complicated and non-relevant, the model can be simplified. In general the cable consists of metallic core and slices of different materials around it, for mathematical correctness tests it is enough to take one slice with typical parameters. The length of a cable (or more cables) is much bigger than its diameter (there are no effects along the cable's length), sand (or soil) area is much bigger than the area of cables, so considering the two-dimensional model in sand is enough. Domain description for mathematical modelling of the problem is presented in Fig. 3. The metal area (with thermal source) is marked with red, sand - grey, isolation blue colour. It has to be noted that coefficient of heat conductivity of isolation is much smaller than that of metal and cables in the whole domain are relatively small $\left(h_{23} \gg d\right)$.

\section{MATHEMATICAL MODELS}

Firs of the all the mathematical models of the problem has to be formulated. The non-stationary problem is given by the parabolic differential equation [6]:

$$
\left\{\begin{array}{c}
c \rho \frac{\partial T}{\partial t}=\sum_{j=1}^{2} \frac{\partial}{\partial x_{j}}\left(\lambda \frac{\partial T}{\partial x_{j}}\right)+q, \quad t \in\left[0, \mathrm{t}_{\max }\right], \quad x \in \Omega, \\
T(x, 0)=T_{b}, \quad x \in \Omega, \\
T(x, t)=T_{b}, \quad x \in \partial \Omega, \\
\sum_{j=1}^{2}\left(\lambda \frac{\partial T}{\partial x_{j}}\right) \text { and } T \text { are continuous, } \quad x \in \Omega .
\end{array}\right.
$$

where:

$x$ is defined as $x=\left(x_{1}, x_{2}\right), T(x, t)-$ function of temperature, $\lambda(x)$ $>0$ - heat conductivity coefficient, $q(x, t, T)$ - the source function, $\rho(x)>0-$ mass density, $c(x)>0-$ specific heat capacity, $T_{b}$ and $t_{\max }$ - constants, $\partial \Omega$ is $\Omega$ domain contour, $\left(x_{1}, x_{2}\right):-L / 2 \leq x_{1} \leq L / 2,-H / 2 \leq x_{2} \leq H / 2$.
Main remarks about the formulated model:

1) $\lambda, \mathrm{c}, \rho$ are discontinuous;

2) values may differ 1000 times, that makes the problem more complicated;

3) $t_{\max }$ can be large, comparing to $T(t)$ dynamics;

4) $\sum_{j=1}^{2}\left(\lambda \frac{\partial T}{\partial x_{j}}\right)$ and $T$ are continuous.

The corresponding stationary problem is given by elliptic differential equation [2]:

$$
\left\{\begin{aligned}
-\sum_{j=1}^{2} \frac{\partial}{\partial x_{j}}\left(\lambda \frac{\partial T}{\partial x_{j}}\right)=q, & x \in \Omega, \\
T(x)=u(x), & x \in \partial \Omega,
\end{aligned}\right.
$$

where:

$$
q(x)=\left\{\begin{array}{ll}
I, & 0 \leq r \leq R_{1} \\
0, & r>R_{1}
\end{array} \text { and } \lambda=\left\{\begin{array}{l}
\lambda_{1}, 0 \leq r \leq R_{1}, \\
\lambda_{2}, R_{1} \leq r \leq R_{2}, \\
\lambda_{3}, r>R_{2} .
\end{array}\right.\right.
$$

but $\lambda_{1}, \lambda_{2}$ and $\lambda_{3}$, are heat conductivity coefficients for metal, isolation and sand, correspondingly.

In turn $R_{1}, R_{2}$ are inner and outer radiuses of isolation ring, while

$R_{3}=\sqrt{2}$ and $r=\sqrt{x_{1}^{2}+x_{2}^{2}}$.

The exact solution of the problem (2) is:

$$
u(x)=\left\{\begin{array}{cl}
\frac{1}{\lambda_{1}}\left(C_{1}-\frac{I r^{2}}{4}\right), & 0 \leq r \leq R_{1}, \\
\frac{C_{2}}{\lambda_{2}}\left(\ln r-\ln R_{1}\right)+C_{3}, & R_{1} \leq r \leq R_{2}, \\
\frac{C_{2}}{\lambda_{3}}\left(\ln r-\ln R_{2}\right)+C_{5}, & r>R_{2} .
\end{array}\right.
$$

where constants are found as:

$$
\begin{aligned}
& C_{1}=-\frac{R_{1}^{2}}{4} I+\lambda_{1}\left[T_{b}-C_{2}\left[\frac{1}{\lambda_{2}} \ln \frac{R_{2}}{R_{1}}+\frac{1}{\lambda_{3}} \ln \frac{R_{3}}{R_{2}}\right]\right], \\
& C_{2}=-\frac{R_{1}^{2}}{2} I, \\
& C_{3}=\frac{1}{\lambda_{1}}\left[C_{1}-\frac{R_{1}^{2}}{4} I\right] \text { and } \\
& C_{5}=\frac{C_{2}}{\lambda_{2}} \ln \frac{R_{2}}{R_{1}}+C_{3} .
\end{aligned}
$$




\section{FINITE VOLUME METHOD}

The problem (1) can be solved utilizing the Finite Volume Method (FVM). Open source software OpenFOAM [14] was used as a base for implementation o FVM calculation schemes. At the same time the authors have developed a $\mathrm{C}++$ independent solver in order to confirm the results of OpenFOAM. All the results presented in this paper were equivalent with both solvers. Discontinuities of coefficients make it necessary to discretize the domain by finite volumes, which would fit to the contours of different sub-domains precisely. So it is necessary to generate special meshes that are adapted to the geometry. However, the complicated geometry of domains with discontinuous coefficients can cause so-called non-orthogonality of a mesh. Additional notes: in this paper only cell-centred FVM approximations are used, harmonic mean formulas for coefficients are necessary (this calculations option is available in OpenFOAM).

\section{A. FVM non-orthogonality error problem solving strategies}

Fig. 4 illustrates non-orthogonality of a mesh of two finite volumes. Non-orthogonality of the mesh and additional error appear, when the vector connecting the centres ( $\mathrm{P}$ and $\mathrm{R}$ ) of neighbouring elements is not collinear to the normal, i.e. $\alpha \neq 0$. It is a well-known problem for non-structured grids, more details can be found, for example, in [15].

Several strategies exist to solve the non-orthogonality problem:

- Correction of the approximation by using iterative methods (supported by OpenFOAM directly).

- Construct dual meshes from the basic Delaunay triangulation.

- Triangulate domain with acute triangles and take Voronoi points as cell centres.

Since the contours of dual mesh finite volumes are not aligned to the contours of domains with different coefficients, the dual mesh construction is more problematic for solution of the problem. That is why it is necessary to consider the first one and the last one presented strategy.

In order to test the efficiency of different solving strategies the same cable was taken as an example. For solving the stationary problem with $T_{\max } \rightarrow \infty, q(\mathrm{x}, t)=q(x)$ it is possible to apply equation (2) and its exact solution. Note that in terms of non-orthogonality it is as weak as original problem, since the non-orthogonality error is magnified by diffusion operator.

The values of other parameters used in calculations:

$\rho_{\text {metal }}=8700, c_{\text {metal }}=385, \lambda_{\text {metal }}=400$;

$\rho_{\text {isolation }}=1380, c_{\text {isolation }}=2000, \lambda_{\text {isolation }}=0.28$;

$\rho_{\text {sand }}=1600, c_{\text {sand }}=890, \lambda_{\text {sand }}=1$.
In case of 3 cables (values of dimensions in [m] from Fig. 3):

$d=0.0348, s=0.0076, H=0.2, L=0.4$;

$h_{1}=0.0826, h_{2}=h_{3}=0.0652$.

For standalone cable in $[\mathrm{m}]$ :

$L=H=0.2$.

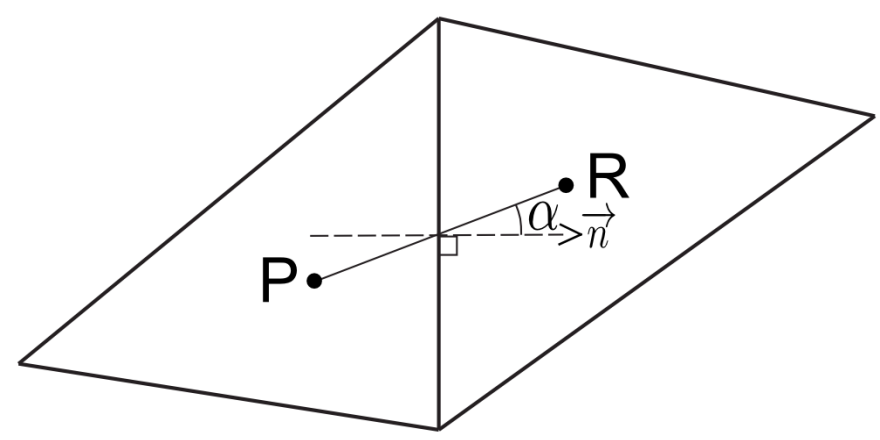

Fig. 4. Non-orthogonality of finite volumes.

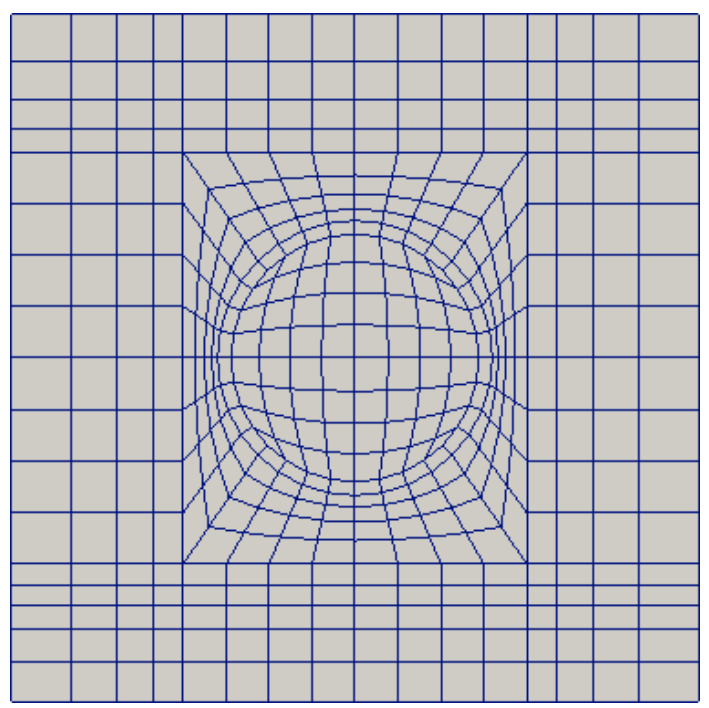

(a)

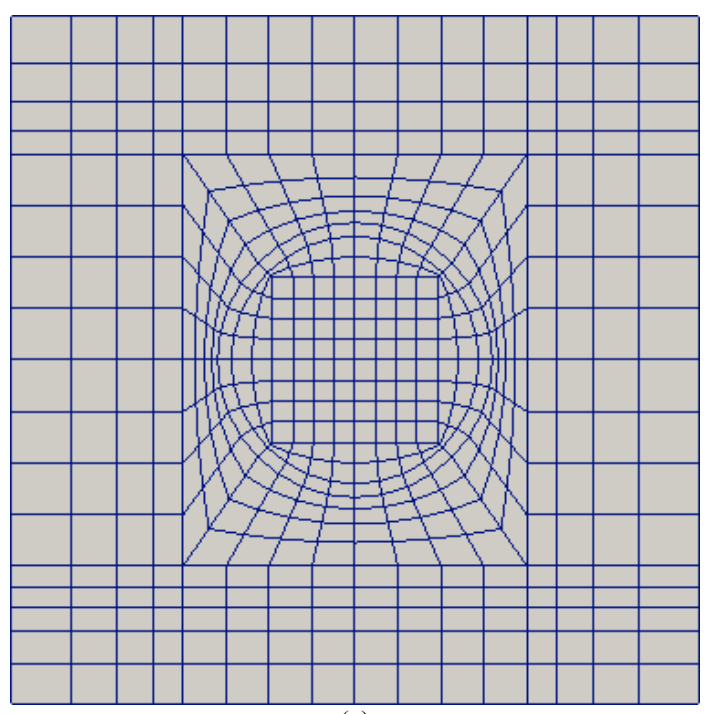

(a)

Fig. 5. Examples of structural meshes. 


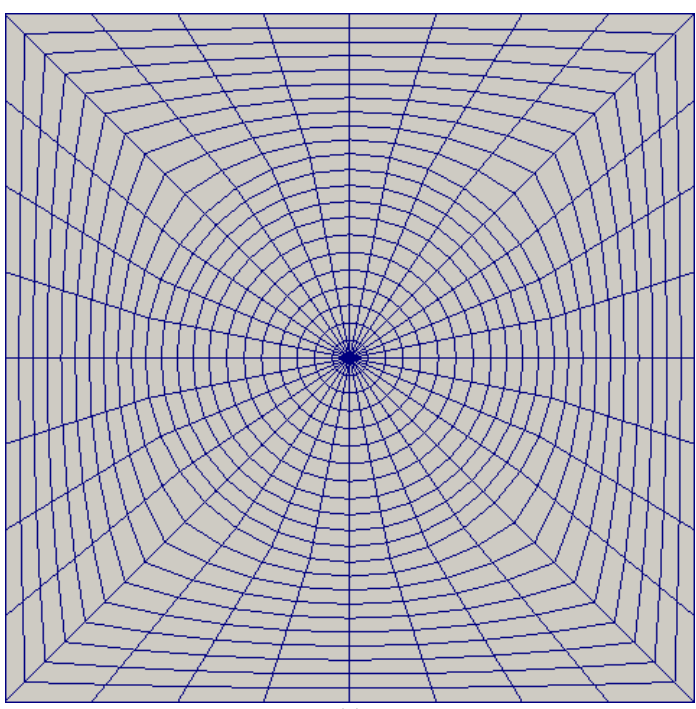

(a)

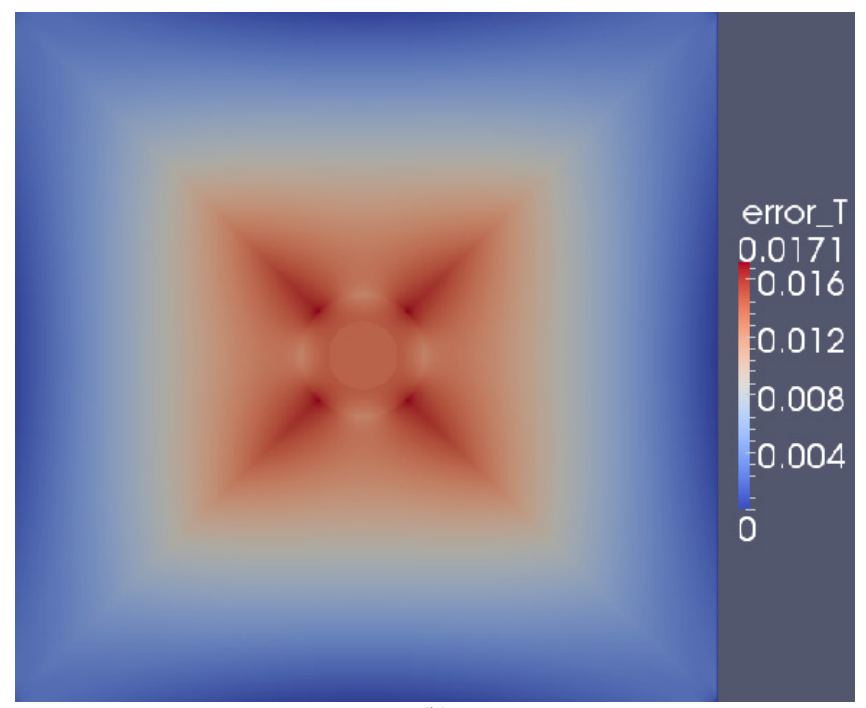

(b)

Fig. 6. Rectangular mesh topology (a) and the corresponding error (b) with $\mathrm{N}=180224$.

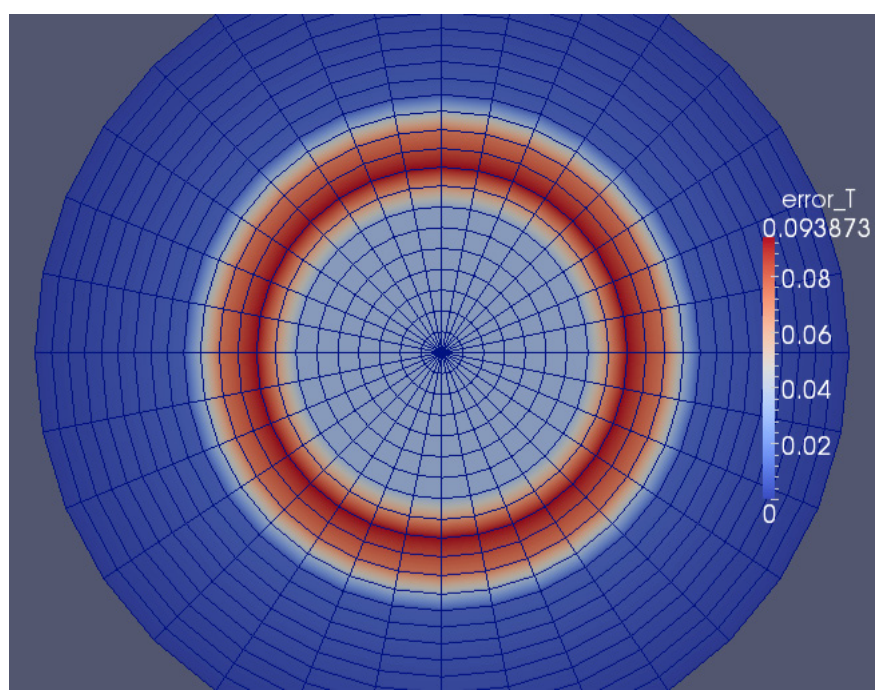

Fig. 7. Error distribution with orthogonal rectangular mesh in C norm.

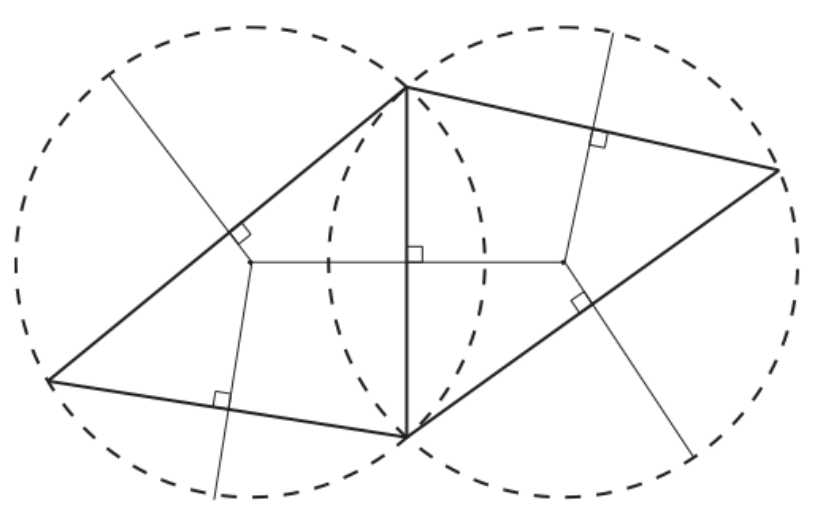

Fig. 8. Mesh orthogonality with acute triangles.

\section{B. Non-orthogonality correction strategy}

The OpenFOAM build-in iterative non-orthogonality corrections approach was tested in the following step. Firstly, the rectangular mesh using OpenFOAM built-in tools was constructed (Fig. 5 and Fig. 6). Then the OpenFOAM built-in non-orthogonality corrections algorithm was tested and the convergence of solution was analysed. The result of this analysis is presented in Table I.

TABLE I

CONVERGENCE FOR NON-ORTHOGONAL MESHES

\begin{tabular}{|c|c|c|}
\hline $\boldsymbol{N}$ & $\boldsymbol{E}_{\boldsymbol{C}}$ & $\boldsymbol{P}$ \\
\hline 704 & 0.27287 & \\
\hline 2816 & 0.143202 & 0.930162 \\
\hline 11264 & 0.071781 & 0.996374 \\
\hline 45056 & 0.035338 & 1.022373 \\
\hline 180224 & 0.017357 & 1.025723 \\
\hline
\end{tabular}

In this table:

$N$ - the number of finite volumes;

$E_{C}-$ defines the error in $C$ norm (maximal difference for all mesh volumes);

$p$ - the experimental convergence order, i.e. $E_{C}=O\left(h^{p}\right)$;

$h$ - the average size of triangles.

It is assumed also that $h=O\left(N^{-p / 2}\right)$. The results presented in Table I show that the convergence rate is lower than theoretical rate of FVM itself $(p=2)$ and non-orthogonality error is dominant. Error distribution is presented in Fig. 6. As it can be seen the error distribution is not radially symmetrical (as the solution is). Just for comparison the error distribution with orthogonal rectangular mesh (without non-orthogonality error) within circular domain is also presented (Fig. 7). This mesh does not fit the analysed case, since boundaries are circular, but the above described problem need to be calculated in a rectangular area.

\section{Discretization with acute triangles}

One more strategy is to use triangular mesh and take Voronoi points as finite volumes centres that form an unstructured orthogonal mesh (Fig. 8). 
This method, however, raises a strict requirement: all angles must be less than 90 degrees. In other words - triangles must be acute. To obtain such kind of meshes the aCute library [16] was used. The corresponding examples of triangulations are presented in Fig. 9.

As it has been mentioned before, cables can be quite far from each other, so it is necessary to generate an adaptive mesh. Adaptive meshes presented in this paper are generated heuristically without any mesh optimality proofs. Let us consider the function of mesh triangle sizes

$$
h(x)= \begin{cases}h_{\text {min }}, & 0 \leq r \leq R_{2}, \\ a r^{\alpha}, & R_{2} \leq r \leq R_{a}, \\ h_{\text {max }}, & r>R_{a} .\end{cases}
$$

where:

$R_{a}$ - the radius of adaptive area; note that $R_{a}>R_{2}$;

$h_{\text {min }}$ - the size of triangles on the contour of the cable;

$h_{\max }-$ the size of triangles in the sand domain.

These parameters are chosen heuristically. At the same time $a$ and $\alpha$ are calculated based on the following requirements:

$$
a R_{2}^{\alpha}=h_{\min } \text { and } a R_{a}^{\alpha}=h_{\max } .
$$

Also for all the experiments $h_{\max }=10 h_{\min }$ and $R_{a}=2 R_{2}$, where $R_{2}$ is the radius of the investigated cable, the adaptive mesh is illustrated in Fig. 10.

The convergence analysis is presented in Table II. First let us make additional notes about experimental convergence rate. Refinement cannot be done uniformly, since mesh must fit coefficients discontinuity contours. Therefore it is not possible to guarantee that in the whole mesh all triangles become smaller in equal proportions. The only way is setting needed parameters for aCute software, so that the refinement is pseudo-uniform. The results presented in Table II show the convergence rate close to 2 .

\section{PARABOLIC PROBLEM SOLUTION}

This section describes the implementation of developed approach to solve the problem (1) with geometry presented in Fig. 3 and parameters presented at the end of subsection IV-A and with backward Euler scheme used for $\partial \mathrm{T} / \partial \mathrm{t}$. The time interval for problem is 24 hours, so that $t \in[0,24]$. The temperature source function is defined as follows:

$$
q(x, t, T)= \begin{cases}g_{0}(T) f_{1}(t), & x \in \Omega_{1, m}, \\ g_{0}(T) f_{2}(t), & x \in \Omega_{2, m}, \\ g_{0}(T) f_{3}(t), & x \in \Omega_{3, m}, \\ 0, & \text { for other } x,\end{cases}
$$

where: $\Omega_{i, m}$ is the metal domain of $i$-th cable (the numeration by index $i$ starts from the left side - see Fig. 3).
TABLE II

CONVERGENCE ACUTE MESHES

\begin{tabular}{|c|c|c|}
\hline $\boldsymbol{N}$ & $\boldsymbol{E}_{\boldsymbol{C}}$ & $\boldsymbol{p}$ \\
\hline 669 & 0.2007691140000 & \\
\hline 2230 & 0.0483012850900 & 2.366660425 \\
\hline 7061 & 0.0149549419800 & 2.034411785 \\
\hline 27639 & 0.0050015730830 & 1.605241562 \\
\hline 110069 & 0.0013158649120 & 1.932524773 \\
\hline
\end{tabular}

Functions are defined as:

$g_{0}(T)=75105(1+0.00393 T)$,

$f_{1}(t)= \begin{cases}1, & t \leq 12, \\ 0, & t>12,\end{cases}$

$f_{2}(t)=\left\{\begin{array}{cr}0.3, & t \leq 6 \\ 1.3, & 6<t \leq 8 \\ 1, & 8<t \leq 18 \\ 1.3, & 18<t \leq 20 \\ 0.3, & t>20\end{array}\right.$

and

$f_{3}(t)=\frac{1}{2}\left(2+\sin \frac{\pi}{4}\right)$.

The functions $f_{1}(t), f_{1}(t)$ and $f_{1}(t)$ are presented graphically in Fig. 11.

The problem was solved with $N=6018$ and with a fixed time step $\tau=0.5 \mathrm{~h}$. The corresponding calculations took 3 seconds with a single core AMD Phenom ${ }^{\mathrm{TM}}$ II X6 1100T 3.3 $\mathrm{GHz}$ processor. The centre of the coordinates of the cables is assigned as $x_{C i}$, where $i$ is the cable number. The corresponding dynamical functions of temperature in the centres of the cables $T\left(x_{C i}, t\right)$ are presented in Fig. 12, Fig. 13 and Fig. 14.

As it is seen from these figures, cables affect each other, despite the fact that the cables are quite far from each other:

- after 20 hours the temperature in the cable 1 becomes slightly lower when the temperature of cable 2 decreases;

- the temperature in the cable 2 slightly decreases, after the source for the cable 1 becomes 0 ;

- the temperature in the cable 3 is lower in local minimum after 22 hours than in local minimum before 20 hours, since the temperature in cable 2 decreases greatly.

Also it is possible to note that after big changes of the source function of the solutions changes fast, so the usage of adaptive time step strategy would be reasonable. However, time-dependent error solving strategy and time step error solving strategy are not discussed in this paper. 


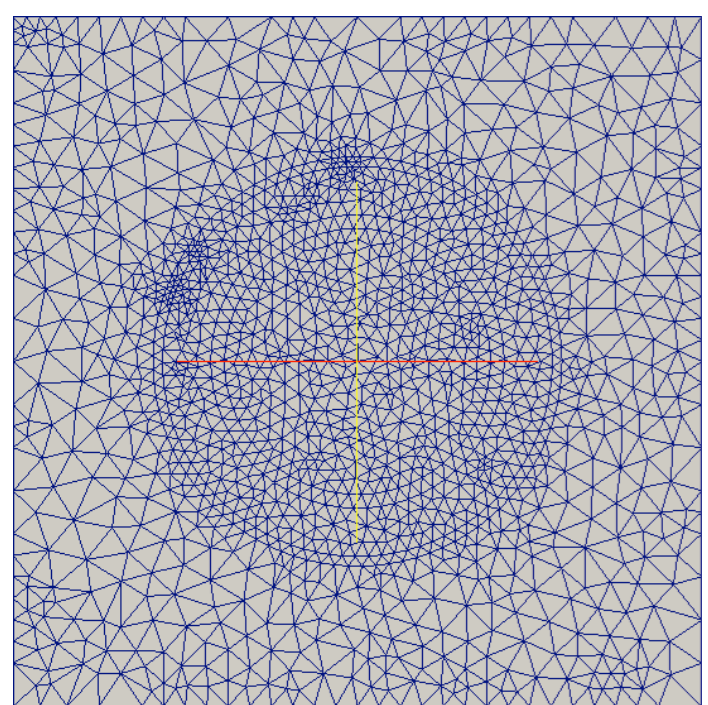

(a)

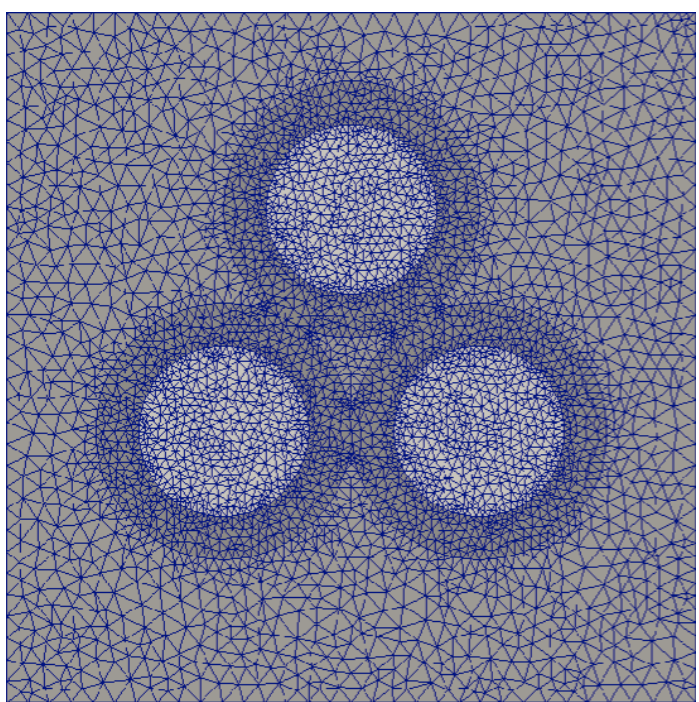

(b)

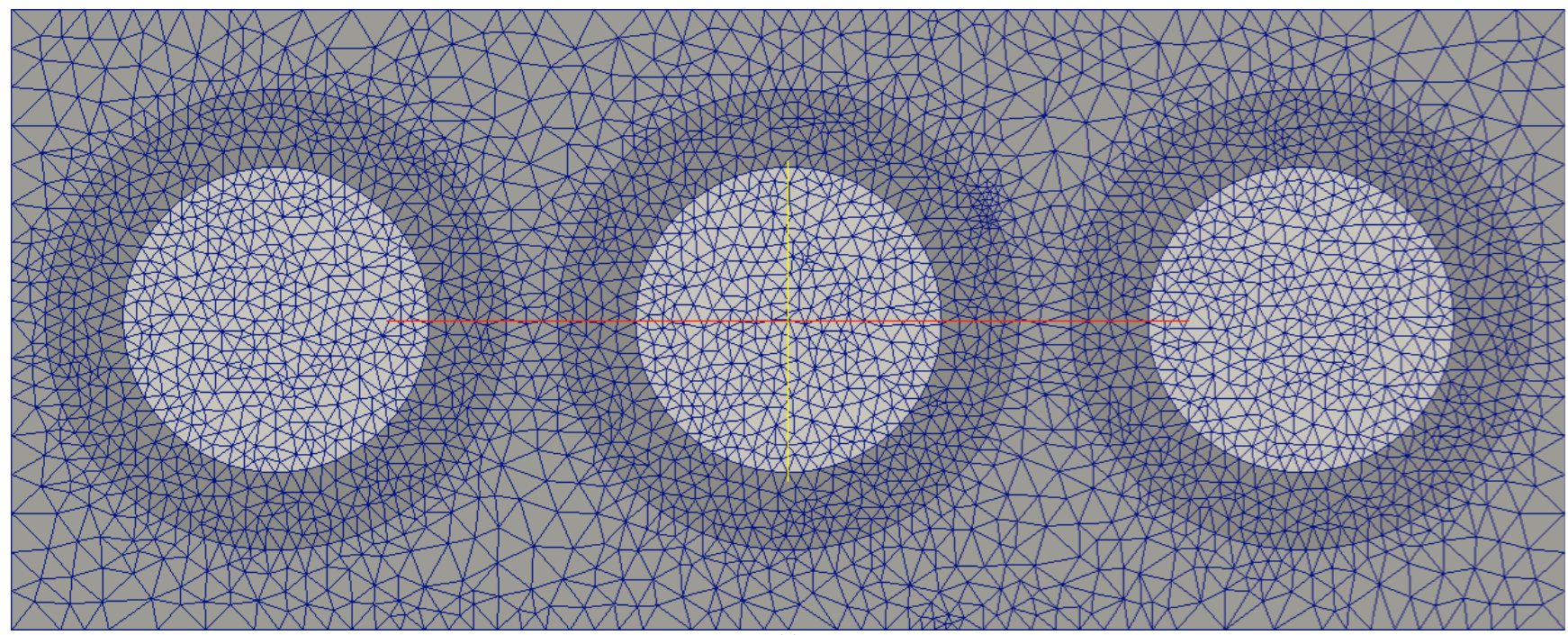

(c)

Fig. 9. Meshes generated with aCute software for (a) stand alone cable, (b) compact triangular placement of three cables, (c) linear placement of of three cables.

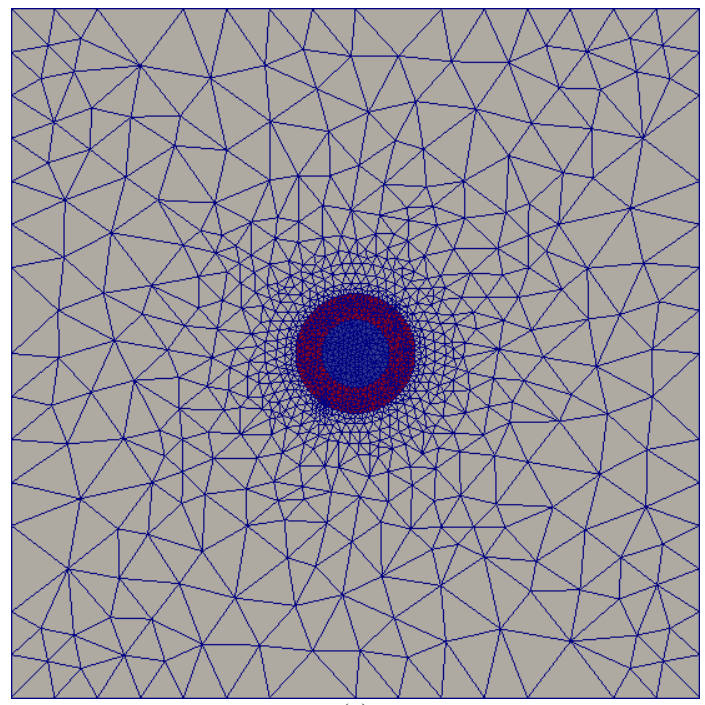

(a)

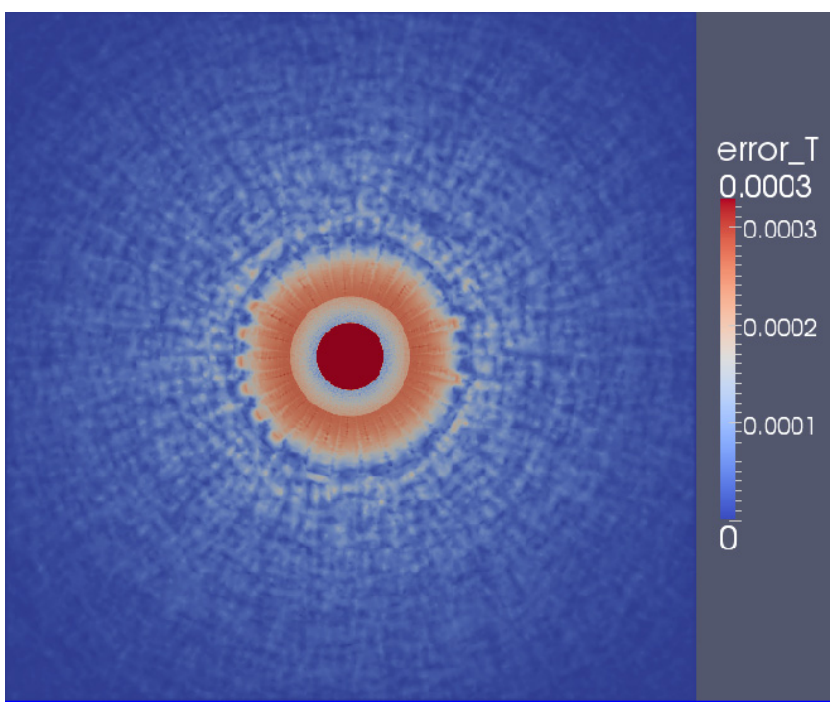

(b)

Fig. 10. Calculations with $\mathrm{N}=435472$ and the mesh topology: (a) mesh topology, (b) error. 


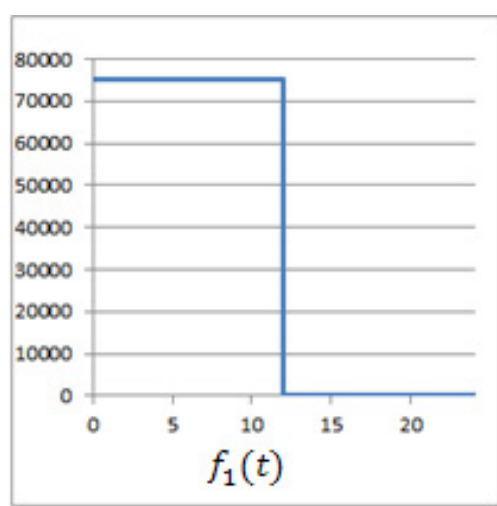

(a)

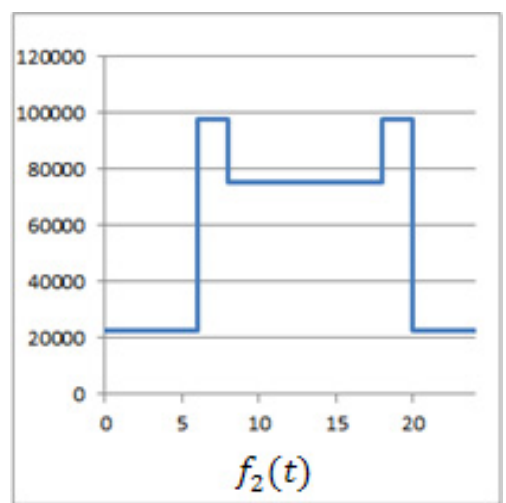

(b)

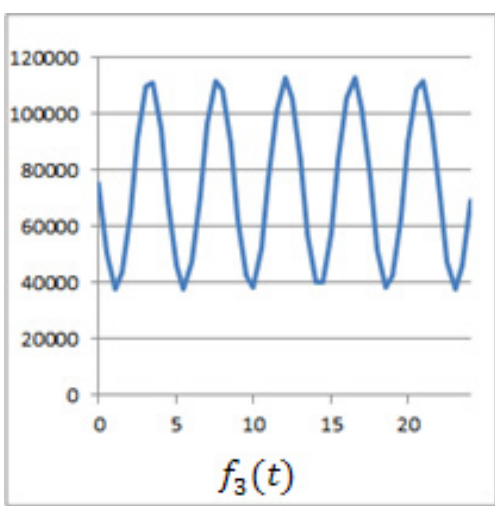

(c)

Fig. 11. Functions for parabolic problem solution: (a) function $f_{1}(t)$, (b) function $f_{2}(t)$, (c) function $f_{3}(t)$

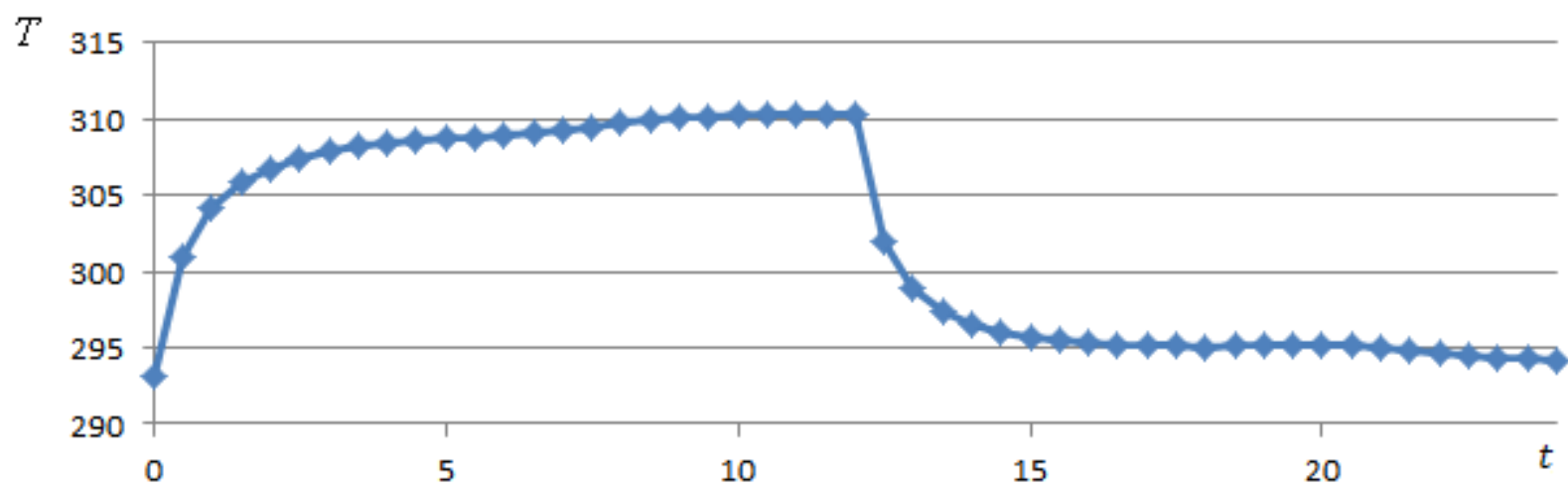

Fig. 12. The temperature in the centre of the first cable $T\left(x_{C l}, t\right)$.

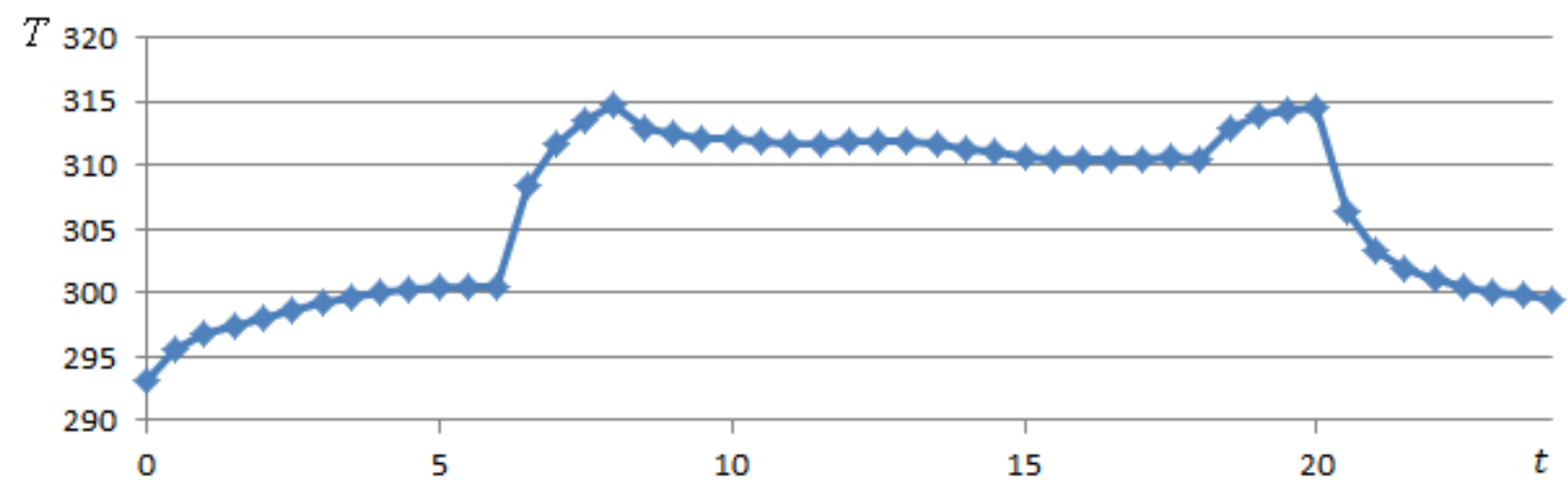

Fig. 13. The temperature in the centre of the second cable $T\left(x_{C_{2}}, t\right)$.

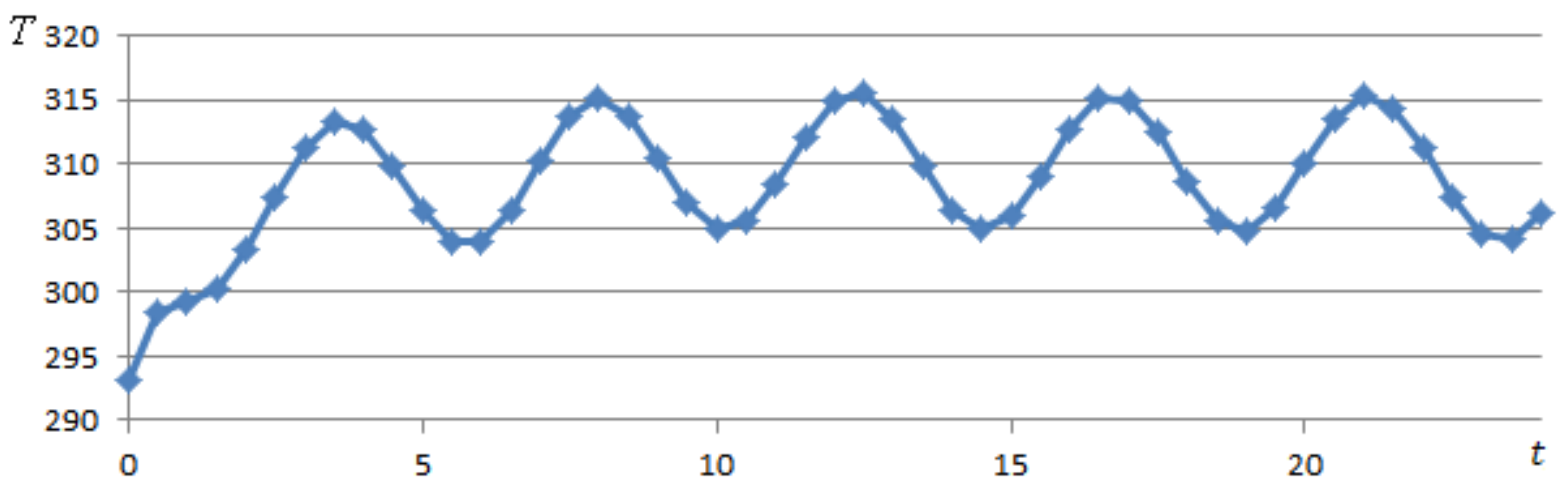

Fig. 14. The temperature in the centre of the third cable $T\left(x_{C 3}, t\right)$. 


\section{Conclusions}

Two different strategies of generating spatial meshes for heat transfer simulation in underground electrical cables were discussed in this paper. Meshes fitting geometry subdomains contours were generated. Usage of rectangular meshes and non-orthogonality corrections methods built-in into OpenFOAM decrease the FVM rate of solution convergence. Domain triangularization with cell centres in Voronoi points eliminates non-orthogonality errors, keeping the FVM order of convergence. For solving real problem spatial adaptive mesh is essential and adaptive time steps also may be required. Heuristic for generating adaptive spatial mesh is proposed. Finally, harmonic mean formulas deal with high coefficients jumps, keeping the error small enough for modelling purposes.

\section{ACKNOWLEDGEMENT}

The work was supported by EUREKA project E!6799 POWEROPT "Mathematical modelling and optimization of electrical power cables for an improvement of their design rules".

\section{REFERENCES}

[1] J. H. Neher, M. H. McGrath. The Calculation of the temperature rise and load capability of cable cystems, AIEE Transactions, Part III, Volume 76, 752-772 p., 1957.

[2] F. Incropera, P. DeWitt, P. David, Introduction to heat transfer. John Willey \& Sons, New Yourk, 1985.

[3] A. Ilgevicius. Analytical and numerical analysis and simulation of heat transfer in electrical conductors and fuses. Dissertation, Universität der Bundeswehr München, 2004.

[4] A. Ilgevicius and H.D. Liess. Calculation of the heat transfer in cylindrical wires and electrical fuses by implicit finite volume method. Mathematical Modelling and Analysis, 8, 217-228, 2003.

[5] J. Taler and P. Duda. Solving Direct and Inverse Heat Conduction Problems. Springer, Berlin, 2006.

[6] R. Čiegis, A. Ilgevičius, H. Liess, M. Meilūnas, O. Suboč. Numerical simulation of the heat conduction in electrical cables. Mathematical modelling and analysis, 12(4), 425-439 p., 2007.

[7] Raim. Čiegis, Rem. Čiegis, M. Meilūnas, G. Jankevičiūtè, V. Starikovičius. Parallel numerical algorithm for optimization of electrical cables, Mathematical modelling and analysis, 13(4), 471-482 p., 2008.

[8] R. Falk and J. Osborn. Remarks on mixed finite element methods for problems with rough coefficients. Math. Comp., 62, 1-19, 1994.

[9] A. Ilgevicius. Analytical and numerical analysis and simulation of heat transfer in electrical conductors and fuses. Dissertation, Universität der Bundeswehr München, 2004.

[10] A.A. Samarskii. The theory of difference schemes. Marcel Dekker, Inc., New York-Basel, 2001

[11] A.N. Tichonov and A.A. Samarskii. Homogeneous finite difference schemes. Zh. Vychisl. Mat. Mat. Fiziki, 1(1), 5-63, 1961.
[12] V.P. Il'in. High order accurate finite volumes discretization for Poisson equation. Siberian Math. J., 37(1), 151-169, 1996.

[13] LeVeque and Z. Li. Erratum: The immersed interface method for elliptic equations with discontinuous coefficients and singular sources. SIAM J. Numer. Anal, 32, 1704, 1995.

[14] http://www.openfoam.com

[15] Loudyi, D. Falconer, R.A. and Lin,B. Mathematical development and verification of a non-orthogonal finite volume model for groundwater flow applications. Adv. Water Res., 30:29-42, 2007

[16] http://www.cise.ufl.edu/ ungor/aCute

Andrej Bugajev has received bachelor (2009) and master (2011) degrees in mathematics. PhD student in Department of Mathematical Modelling, Vilnius Gediminas technical university. Andrej works at the Department of Mathematical Modelling of Vilnius Gediminas Technical University since 2011. Research interests: theory of algorithms, numerical methods, parallel computing, computing using GPU, mathematical modelling.

Postal Address: Department of Mathematical Modelling, Sauletekio av. 11, LT-10223 Vilnius, Lithuania

E-mail: andrej.bugajev@vgtu.lt

Gerda Jankevičiūtè has received Bachelor of Science in Applied Mathematics, Faculty of Fundamental Science, Kaunas University of Technology, 2001. Master of Science in Applied Mathematics, Faculty of Fundamental Science, Kaunas University of Technology, 2003. Doctor of science in Mathematics, Vilnius Gediminas Technical University. Ass. Professor at Mathematical Modelling department in Vilnius Gediminas Technical University, September 2010 to present.

Postal Address: Department of Mathematical Modelling, Sauletekio av. 11, LT-10223 Vilnius, Lithuania

E-mail: gerda.jankeviciute@vgtu.lt

Olga Suboč has received Bachelor (1996) and Master (1998) degrees in mathematics, Vilnius University, Faculty of mathematics and informatics. Doctor degree in physical sciences, mathematics (2003, VGTU, MII).

From 1998 works in Vilnius Gediminas technical university, Department of Mathematical Modelling, from 2003 till now in position of assoc. prof. In 2009 received diploma of associated professor in physical sciences. Research interests: numerical methods, mathematical modelling, discrete mathematics, non-local conditions.

Member of Lithuanian Mathematical Society.

Postal Address: Department of Mathematical Modelling, Sauletekio av. 11, LT-10223 Vilnius, Lithuania

E-mail: olga.suboc@vgtu.lt

Natalija Tumanova has obtained $\mathrm{PhD}$ degree in mathematics in 2012. The field of studies covered numerical analysis of nonlinear models in nonstandard domains and domain decomposition methods for parallel computing.

N. Tumanova works at the Department of Mathematical Modelling of Vilnius Gediminas Technical University since 2007, in the capacity of Associated Professor and Researcher since receiving her PhD in 2012. The main fields of research interests are mathematical modelling, numerical analysis of PDE and parallel computing.

Postal Address: Department of Mathematical Modelling, Sauletekio av. 11, LT-10223 Vilnius, Lithuania.

E-mail: natalija.tumanova@vgtu.lt 